\title{
Interfacial Colloidal Performance and Adhesive Strength of an Environmentally Friendly Cellulose-microcrystal-based Adhesive Substance
}

\author{
Zhexu Xi ${ }^{1,}$,, Jianfei Feng ${ }^{2}$, Xiaolin $\mathrm{Lu}^{2}$ \\ ${ }^{1}$ Bristol Centre for Functional Nanomaterials, University of Bristol, Bristol, UK \\ ${ }^{2}$ College of Materials, Xiamen University, Xiamen, China
}

Email address:

hu20363@bristol.ac.uk (Zhexu Xi),3408147197@qq.com (Jianfei Feng), 1718347737@qq.com (Xiaolin Lu)

${ }^{*}$ Corresponding author

To cite this article:

Zhexu Xi, Jianfei Feng, Xiaolin Lu. Interfacial Colloidal Performance and Adhesive Strength of an Environmentally Friendly Cellulose-microcrystal-based Adhesive Substance. American Journal of Nanosciences. Vol. 6, No. 4, 2020, pp. 24-33. doi: $10.11648 /$ j.ajn.20200604.11

Received: December 16, 2020; Accepted: December 29, 2020; Published: January 12, 2021

\begin{abstract}
As one of the high-value and environmentally friendly chemical products, cellulose-based adhesive substance has been widely developed recently due to its extraordinary adhesion, interfacial flowability and high stability, along with low-impact side effects, outstanding degradability and biocompatibility. Also, microcrystalline-shaped cellulose has been fully exploited in functinalizing modification engineering. To eliminate the gap in application of cellulose-ether-based adhesion, we fabricated a new category of optimized cellulose sulfate product based on a system of analysis of key experimental factors: solvent pretreatment, degree of esterification, type and morphology of cellulose. Additionally, we focused on an all-round family of performance test of our product, containing characterization of structural and morphological features, test of water-based properties and inspection of adhesion behaviour. Within, the effect of adhesive strength in various solvents and under various conditions have been highlighted. The whole result shows a generally feasible synthetic approach and splendid performance as a novel green adhesive. The optimal design and synthesis of cellulose sulfate is verified to be eco-friendly, easy for optimization and greatly reproducible. Also, the product is shown to have tiptop viscosity, great adhesive strength, and colloidal potential like film forming due to the cross-linking network in the water-based environment. Finally, the product are demonstrated to be outstanding and widely applicable in mechanical strength. Specifically, it shows strong adhesion on different materials (metal, timber and glass) and tunability related to the kinds of additives. The addition of metallic salts and polymeric amine can evidently boost its adhesive strength. Overall, optimal analysis of cellulose sulfate adhesive on a microscale has forecasted great potential in low-carbon, fine-tunable, adhesive-related industry.
\end{abstract}

Keywords: Cellulose Sulfate, Adhesion, Viscoelasticity, Colloidal Behavior, Hydrodynamic Performance, Characterization, Cross-Linking, Environmentally Friendly

\section{Introduction}

With the booming of adhesive technology, adhesive with ideal adhesive performance has broad applicability in many fields, like aerospace, building materials, medical care and timber processing [1]. With its increasing market capacity, a new kind of environmentally friendly and high value-added adhesive product becomes the heated mainstream for the goal of less pollution, lower side effects and sustainability. Accordingly, to highlight, an infinitely superior adhesive product should be designed and synthesized in green, cleaning experimental system.

On the basis of this standard, also known for outstanding compatibility and environmental affinity, cellulose-functionalized adhesive is deemed as a perfect substitute for polluted conventional one [2]. Besides its abundant reserves and wide distribution in nature, cellulose have potential in modification and exploitation due to its strong hydrophilic free hydroxyl and reactivity [2-4]. Moreover, its structure plays a double-edge-triggered role: 
the ordered sequence alignment and tight hydrogen bonding facilitate its chemical stability and well-regulated pattern; its tight-arranged, highly-crystalline chain conversely hinder the soluble dispersion and reactivity because of possibilities in particle agglomeration and random-coiled cellulose with higher crystallinity $[4,5]$.

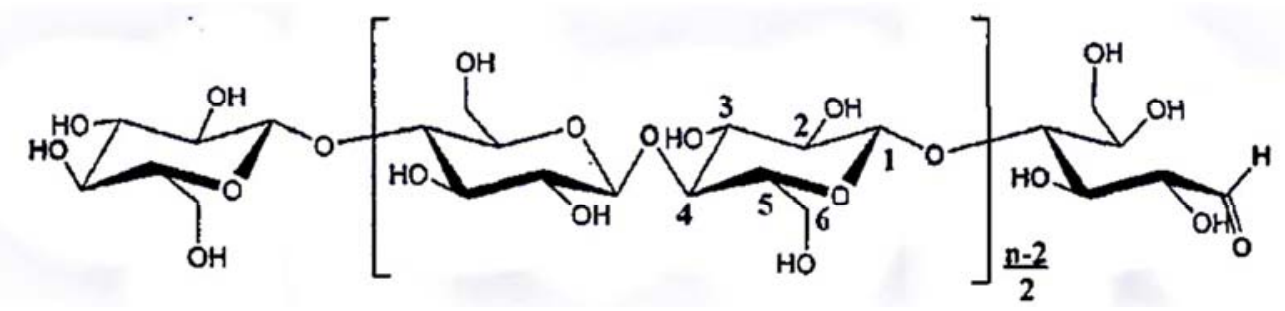

Figure 1. Chair conformation of the cellulose structure.

To strengthen the reactivity of cellulose, proper modified derivatization strategies need to be taken into implementation. Within, one method aims at the crystalline of cellulose, regarding macrolevel shape and microlevel morphology, such as thin-film-oriented and microsphere-related modification [6, 7]. Another focuses on the chemical modification of free hydroxyl groups of cellulose for more specific properties [8]. Here, esterification of cellulose was chosen as a modified adhesive in place of traditional etherification approaches [4, 7].

The reason for proposed cellulose sulfate (CS) as a potential product is enhancing solvent accessibility by undermining hydrogen bonding. Meanwhile, a number of additional extraordinary properties are generated with the functionalizing hydroxyl. It has a markedly concentration-sensitive viscosity, which is similar to that in a polymer solution [8]. Also, its share-resistant performance, suspension stability and film-forming property are all intimately tuned with esterification degree [7-9]. Its great compatibility with metal ions can dramatically increase the adhesive strength [9]. More vitally, these adhesion-related properties can be inspected, analyzed and well regulated in our experiment.

Finally, the question is, why we sustain an extra focus on the adhesive strength and colloidal performance of the product? First, identify the role of rigid chain and flexible functionalized hydroxyl in CS. Cellulose-based products all have a rigid linear chain, which clarifies the stress-strain correlations. This entails an index-apparent viscosity. On the other hand, esterifcation strategy leads to a more hydrophilic interface with more easily modulated characteristics. Thus, the molecular system has simultaneously modulus of elasticity (also known as flexibility) and rigidity, which contributes differently. Second, how does the viscoelasticity of CS affect the interfacial morphlogical feature as well as the sol-gel transition at interface? Two main factors regarding the viscoelastic behaviour in the compressive polymer-solvent system refer to the hydrodynamic size of the single molecule for the low-concentration solution and of the polymer induced by intermolecular and intramolecular interaction for the high-concentration. The key to this transition is a tunable association or crosslinking, other than purely increasing the molecular weight, which is the powerful evidence for the regulation of viscosity and flexibility. Third, how does this interfacial modulation affect adhesion? As a water-based dispersion system, CS adhesive generates a cross-linking network where hydrophilic residues makes the inner water-soluble and rigid chains makes the outer swelling polymers $[1,8,9]$. This linking capacity can directly reveals the macroscale adhesive strength. This controllable phase transition depends entirely on the degree of cross-linking, which signifies the relative two-phase flow at solvent-polymer interface.

Hence, in this paper, for the aim of the optimal design of a novel environmentally friendly adhesive product and further the correlations between colloidal-related adhesion and its inner mechanism, we propose CS as the ingredient of our new product with an all-around optimization experiment. Also, we focus on the characterization and test of the product, including structure and morphology, adhesion-related properties and the factors. Especially, we highlight the connections between interfacial adhesive capacity and its hydrodynamic polymerization.

\section{Results and Discussion}

\subsection{Characterization of Product}

\subsection{1. ${ }^{13}$ C-NMR Spectral Characterization}

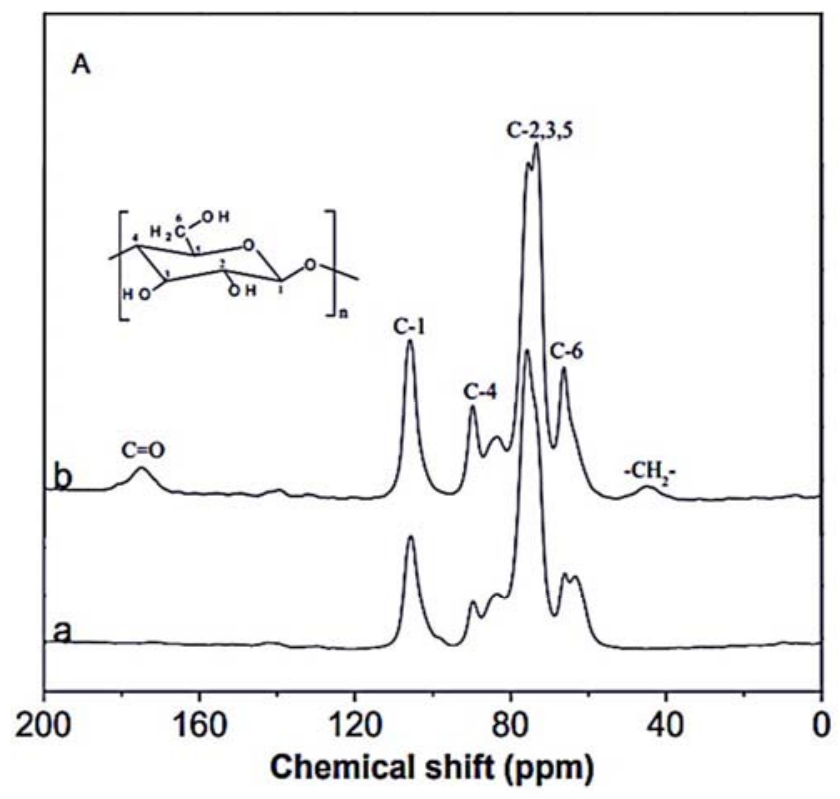

Figure 2. The ${ }^{13} C$-NMR spectrum of cellulose. 


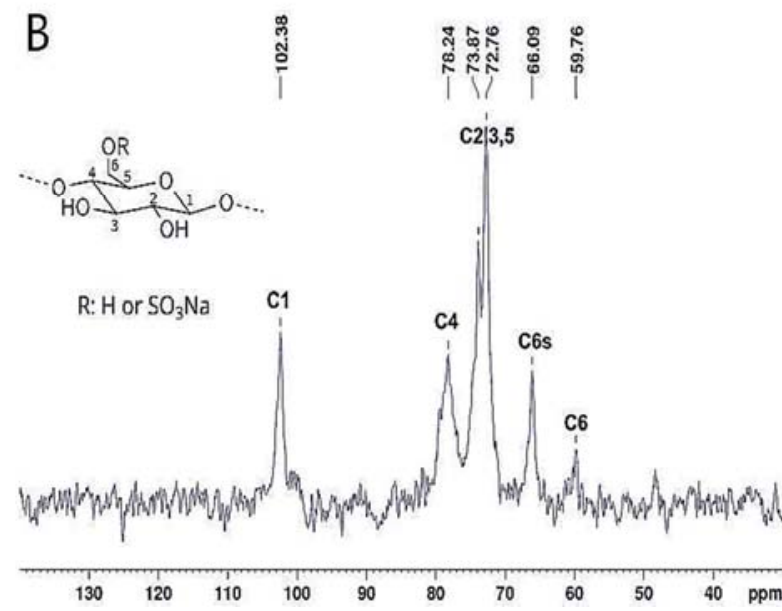

Figure 3. The ${ }^{13} C$-NMR spectrum of cellulose sulfate.

As displayed in the ${ }^{13} \mathrm{C}$-NMR spectrum, a new peak at $65.91 \mathrm{ppm}$ was observed for sodium cellulose sulfate, compared with cellulose. It is the result of the substitution of primary hydroxide radical at $\mathrm{C} 6$ with sulfate group. The peak for unsubstituted C6 hydroxide radical was located at 60.00 ppm. Since both peaks are associated with the C6 signal, the degree of substitution on $\mathrm{C} 6$ can be obtained by the integration of the two peaks. The signal for $\mathrm{C} 3$ is located at around 79 ppm. The degree of substitution on $\mathrm{C} 3$ can be obtained by the difference between total degree of substitution and the degree of substitution on C2 and C6. Due to the influence of sulfate groups, the chemical shifts of C5 and C6 are slightly changed in sodium cellulose sulfate compared with cellulose. In summary, the product is mainly C6 substituted sodium cellulose sulfate. The degree of substitution calculated for the product is 0.46 .

Due to the inductive effect and anisotropy effect caused from the substitution of hydroxide radicals at certain carbon position, a strong electron-donating effect was observed, resulting in a left shift of the carbon formant and a varying degree of low field displacement [10]. The exact value depends on the spatial conformation of cellulose sulfate, strength of shielding effect and the electron cloud density. It displayed a rather weak peak for C6 position. An absorption peak at $65.91 \mathrm{ppm}$ near the high field was observed, which could be the result of the substitution of most hydroxide radicals by sulfate groups and the formation of strong C6 sulfate. The peak for $\mathrm{C} 2$ was weak while a strong peak located at around $89.6 \mathrm{ppm}$ was observed, which could be the substitution of $\mathrm{C} 2$ hydroxide radicals by sulfate groups. The shift on C2 is larger than that on C6. That's probably because C2 had C3 hydroxide radicals around it, thus forming an electron-donating effect, enhancing the electron cloud density, and finally leading to stronger shielding effect. It indicates that the possibility for hydroxide radicals in cellulose substituted by sulfate groups is $\mathrm{C} 6>\mathrm{C} 2>\mathrm{C} 3$. It is in accordance with the reference. Steric hindrance also has an influence on the order and degree of substitution. As can be seen from the free hydroxide radicals, the primary hydroxide radicals attached to C6 have the highest activity. The secondary hydroxide radicals attached to $\mathrm{C} 2$ is less active than the primary hydroxide radicals. Due to steric-hinerance effect, the hydroxide radicals at $\mathrm{C} 3$ have the lowest activity for substitution. Therefore, we suggest that the cellulose sulfate obtained was substituted at C6 and C2, and C6 is the main component. The substitution at $\mathrm{C} 3$ will only happen when the degree of substitution is high. As shown in the spectrum, a new peak could be observed around the high field of $\mathrm{C} 1, \mathrm{C} 4$ and C5. That's probably because the shielding effect occurs after the substitution of $\mathrm{C} 2$ and $\mathrm{C} 6$ hydroxide radicals by sulfate groups, resulting in the de-shielding effect on the carbon nearby, and lowering electron cloud density. Thus, a shift was observed at the peak located at about $104.6 \mathrm{ppm}$, $82.3 \mathrm{ppm}$ and $72.0 \mathrm{ppm}$ which is the result of $\mathrm{C} 1, \mathrm{C} 4$ and $\mathrm{C} 5$.

\subsubsection{FTIR Spectral Characterization}

The figure below shows the FTIR spectra of cellulose and cellulose sulfate with different degree of substitution. The peak located at $3600-3200 \mathrm{~cm}^{-1}$ is the vibration mode of $-\mathrm{OH}$. The peak at $2900 \mathrm{~cm}^{-1}$ is the stretching mode of $-\mathrm{OH}$ attached to $-\mathrm{CH}_{2}$. The two peaks become broader and weaker as the degree of substitution increases. This suggests that some of the hydroxide radicals were substituted, further indicating that the content of C6 substitution increased. The peak at $1240 \mathrm{~cm}^{-1}$ is the result of $\mathrm{S}=\mathrm{O}$, while the peak at $850-820 \mathrm{~cm}^{-1}$ is associated with the $\mathrm{C}-\mathrm{O}-\mathrm{S}$ stretching mode vibration. New absorption peaks were observed in b-e indicating the appearance of $-\mathrm{SO}_{3}$ groups. It is further evidence for the substitution of $-\mathrm{OH}$ on cellulose by $-\mathrm{SO}_{3} \mathrm{Na}$. Different peak intensity indicates different position and degree of substitution. In addition, the possibility for the substitution of $\mathrm{C} 6$ becomes higher as the degree of substitution increases.

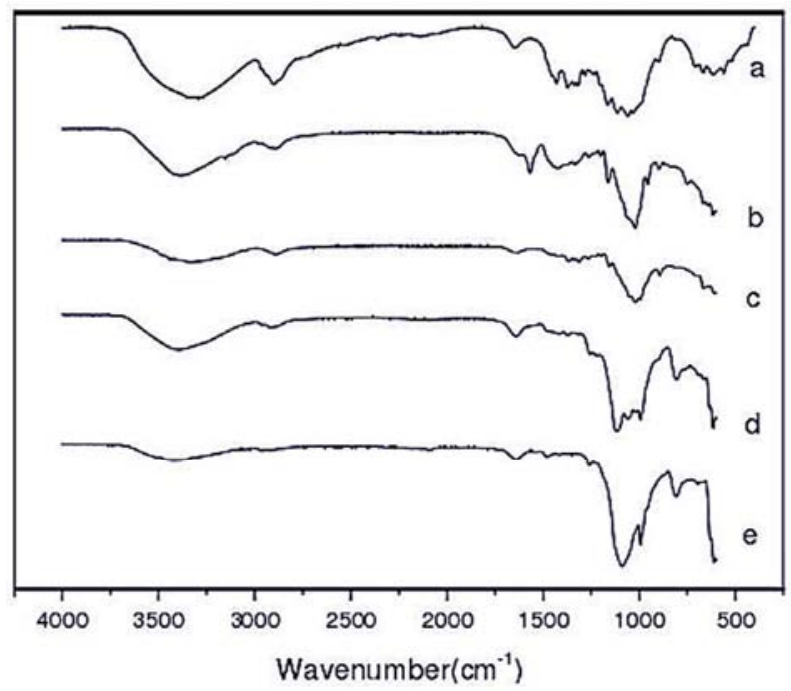

Figure 4. A series of IR spectrum of cellulose sulfate: a) is cellulose as a raw material, and b) to e) shows information on $C S$ in substituting degree $0.27,0.48,0.61$ and 0.99 .

\subsubsection{X-ray Diffraction Analysis}

XRD analysis was applied to characterize the crystal structure of cellulose sulfate [11]. In order to eliminate the influence of the crystal orientation, cellulose sulfate was 
made into film $[3,12,13]$.

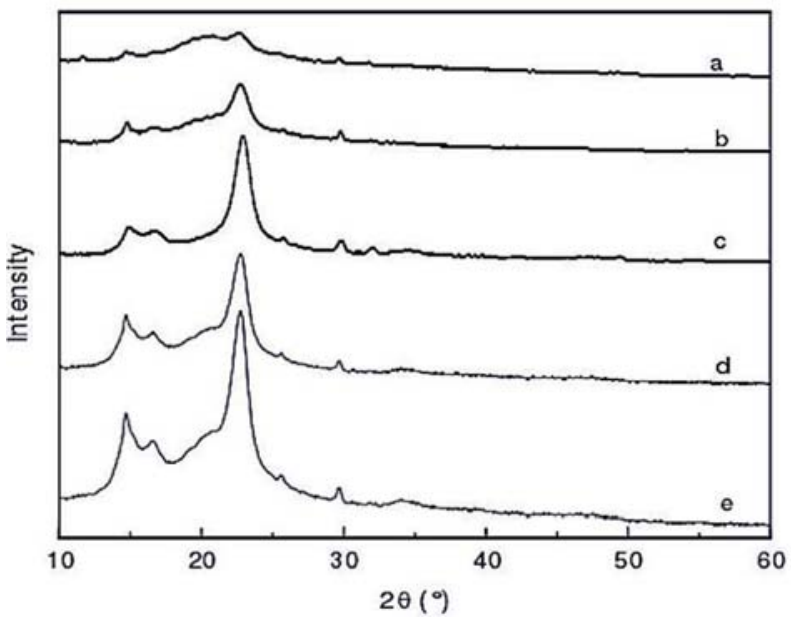

Figure 5. A series of XRF spectrum where a) to d) are cellulose sulfates in substituting degree 0.99, 0.61, 0.48 and 0.27, and e) is cellulose.

The diffraction peaks located at $2 \theta=15.1^{\circ}$ and $22.6^{\circ}$ are the typical characteristic peaks of type I cellulose. From the above figure, it was obvious that CS0.27 and CS0.48 have strong diffraction peaks at $2 \theta=15.1^{\circ}$ and $22.6^{\circ}$, indicating that CS, with lower degree of substitution ( $\mathrm{DS}=0.2 \sim 0.5)$, has better crystallinity. While the diffraction peak for CS0.61 is greatly weakened, indicating that the crystalline structure of CS is broken when the degree of substitution is greater than 0.6. The diffraction peak for CS0.99 is rather weak, indicating that CS displays an amorphous structure when the degree of substitution reaches 1.0 or higher. The reason may be that cellulose has rigid structure, in which the molecular arrangement is dense and neat. While cellulose sulfuric acid ring ester, which is the intermediate product of sulfuric acid esterification process, has larger intermolecular distance, resulting in weaker van der Weal forces and lower crystallinity. Besides, the introduction of phosphoric ester would break the intermolecular hydrogen bonds and destroy its stable linear structure, which leads to disturbed molecular arrangement, extended molecular chain and destroyed crystalline structure. As the amount of esterifying agent $\mathrm{ClSO} 3 \mathrm{H}-\mathrm{DMF}$ increases or the esterification time prolongs, the hydrogen-bond interaction between molecules becomes weaker, the molecular arrangement gets disordered, thus resulting in lower crystallinity of CS.

The above results indicate that cellulose is not completely substituted in the process of sulfuric acid esterification reaction. Therefore, the product with low degree of substitution display strong diffraction peaks while products with high degree of substitution undergo a more complete reaction process. It further indicates that cellulose with high degree of substitution ( $\mathrm{DS} \geq 0.99$ ) is amorphous. It is because that the introduction of sulfate groups in the esterification process would destroy the hydrogen bond inside and between the cellulose molecules and broke the neat crystal structure of cellulose. It indicates that the cellulose sulfate with high degree of substitution displays a lower crystallinity.

\subsubsection{Differential Scanning Calorimetry (DSC) Analysis}

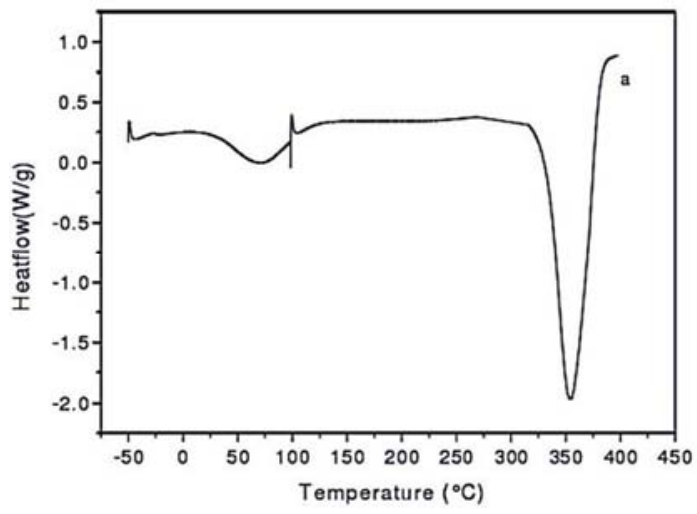

Figure 6. DSC curve for cellulose.

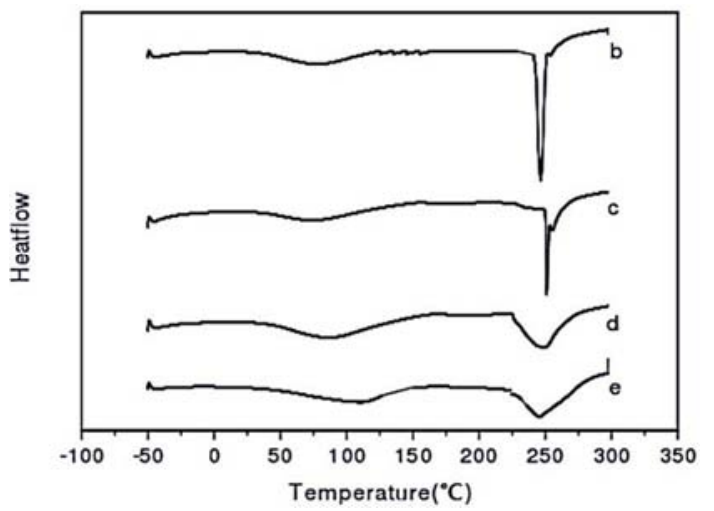

Figure 7. DSC curves for b CS0.27, c CS0.48, $d$ CS0.61 and e CSO.99.
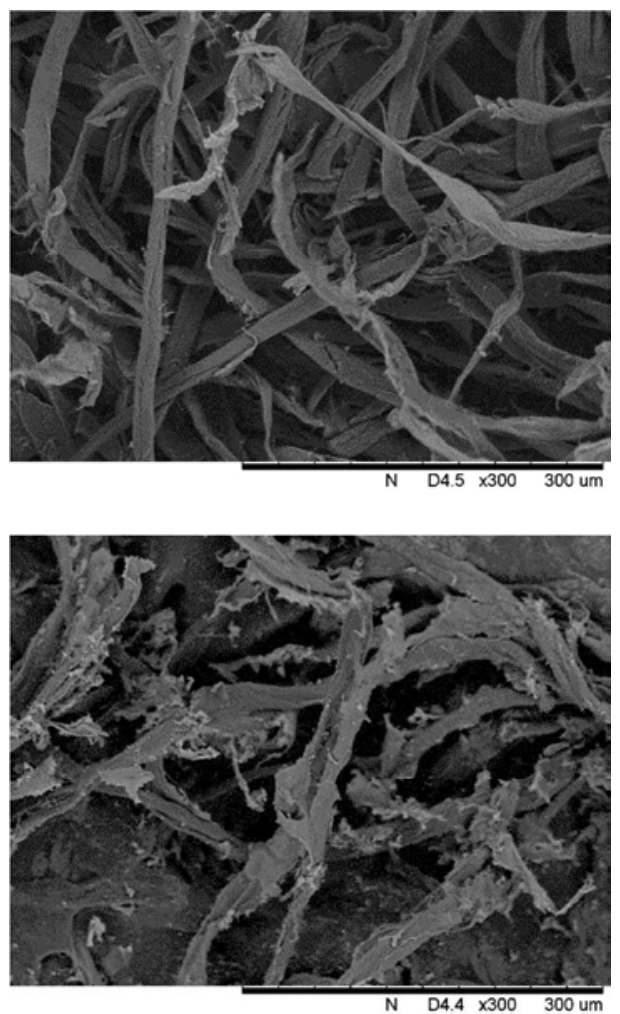

Figure 8. SEM images for cellulose (up) and CS (down). 
As seen from the FTIR spectra, cellulose sulfate possesses the crystal structure of type I cellulose, but in lower crystallinity compared with cellulose. As shown in Figures 1 and 2, cellulose displays a broad endothermic peak at $72^{\circ} \mathrm{C}$, resulting from the decomposition of bound water in the cellulose molecules. As the degree of substitution of cellulose increases, the decomposition temperature for the bound water becomes higher. The decomposition temperature is $76,77,90$ and $112^{\circ} \mathrm{C}$, respectively as the degree of substitution increases. It mainly results from the low crystallinity of cellulose sulfate and the hydroxide radicals and sulfate groups in the structure, which can form hydrogen bound with water. In addition, sulfate groups possess strong hydrophilicity and better water conservation capability. Thus, it requires a higher temperature for the decomposition of bound water in the cellulose sulfate. As can be seen from Figure 1, the endothermic melting peak of cellulose is located around $353^{\circ} \mathrm{C}$. While the introduction of sulfate groups caused a shift in the endothermic melting peak of cellulose sulfate, compared with cellulose. The melting point of cellulose sulfate is lower than that of cellulose. This is because the main molecular chain of cellulose and cellulose sulfate was broken and decomposed into small molecules or glucose under high temperature. Thus, the melting peaks of the cellulose sulfate display at $250,253,252$ and $245^{\circ} \mathrm{C}$, respectively as the degree of substitution increases. It indicates that cellulose sulfate possess lower thermal stability than cellulose, and the melting point decreases when the degree of substitution increases. This results from the fact that the substitution of hydroxide radicals by sulfate groups in the esterification process weakened the hydrogen bound inside and between the molecules in the system. The rigid chain segment structure of the molecule was destroyed. The arrangement of the molecules becomes disturbed. Some of the molecules are even degraded and the molecule structure become unstable, which benefits the dissolve and formation of hydrogels.

\subsubsection{Scanning Electron Microscope (SEM) Analysis}

The SEM images indicate that cellulose possess a fiber-shaped morphology with a diameter of 100-300 mm while the cellulose sulfate displays a ribbon-like structure with rough surfaces which demonstrating the destroy of structures. The hydrogen bound inside and between the molecules was destroyed in the sulfuric acid esterification process, resulting in great change in morphology and decreased crystallinity. Therefore, the products have better water solubility and can form hydrogels more easily.

\subsubsection{Measurement of Other Parameters}

a. Calculation of the degree of substitution

According to the equation described as

$$
\mathrm{DS}=\frac{\mathrm{S} \% / 32}{\mathrm{C} \% / 72}=2.25 . \mathrm{S} \% / \mathrm{C} \%
$$

The corresponding results are shown in the table below.

Table 1. The result of sample's degree of esterification.

\begin{tabular}{lllllllll}
\hline Sample & $\mathbf{C} \%$ & N\% & H\% & S\% & C/N & H/C & D $/ \mathbf{S}$ & \\
\hline $\mathrm{NaCS}$ & 24.88 & 0.32 & 3.57 & 10.51 & 78.14 & 1.72 & 6.31 \\
\hline
\end{tabular}

\section{b. Measurement of the molecular weight}

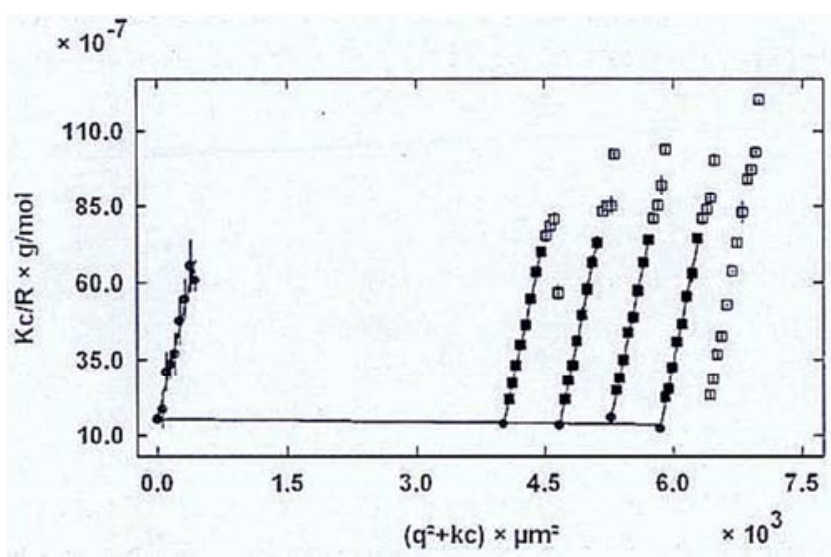

Figure 9. The Debye plots whose model precisely evaluate and judge the molecular weight.

According to the information from light scattering database, the molecular weight calculated was 550 thousand.

\subsection{Analysis on the Experimental Conditions}

The samples were characterized according to the amount of chlorosulfonic acid, the pretreatment of solvent and the morphology of products $[2,14]$. The accidental error caused by the experimental operation and equipment could be ignored. Different control variates were used to guarantee the minimum error. The conclusion was reached by controlling the three different varieties, setting a series of variety and repeating the mentioned characterization.

\subsubsection{The Explanation to the Pretreatment of Solvents}

Little molecules can only reach $10-15 \%$ of the hydroxide radicals in cellulose with high crystallinity. It is widely believed that most regents can only reach the amorphous region which is also called the accessible region of cellulose but not the neat crystalline zone. The pretreatment of cellulose by solvents can induce profoundly change in the polymerization structure [15-17]. For instance, the lowered crystallinity, decreased size of microcrystal, increased micro-pores, lowered degree of polymerization and broken hydrogen bond inside and between the molecules is often observed. Cellulose molecules inside the chain-sheet are hydrophobic, while the hydroxide radicals between the chain-sheets are hydrophilic. When cellulose is pretreated with solvents, the reaction unit is chain-sheet but not a single molecule 
chain. The solvents are intercalated from the gap between the chain-sheets and break the hydrogen bond between the sheets, resulting in the exposure of hydroxide radicals. Thus, the accessibility of regents is improved.

The longer the pretreatment maintains, the shorter the reaction time gets. It is due to the long pretreating time benefits the intercalation of solvents, leading to more broken hydroxide radicals. Therefore, the accessibility of chlorosulfonic acid is greatly improved. Hence, the soaking should be maintained more than one day.
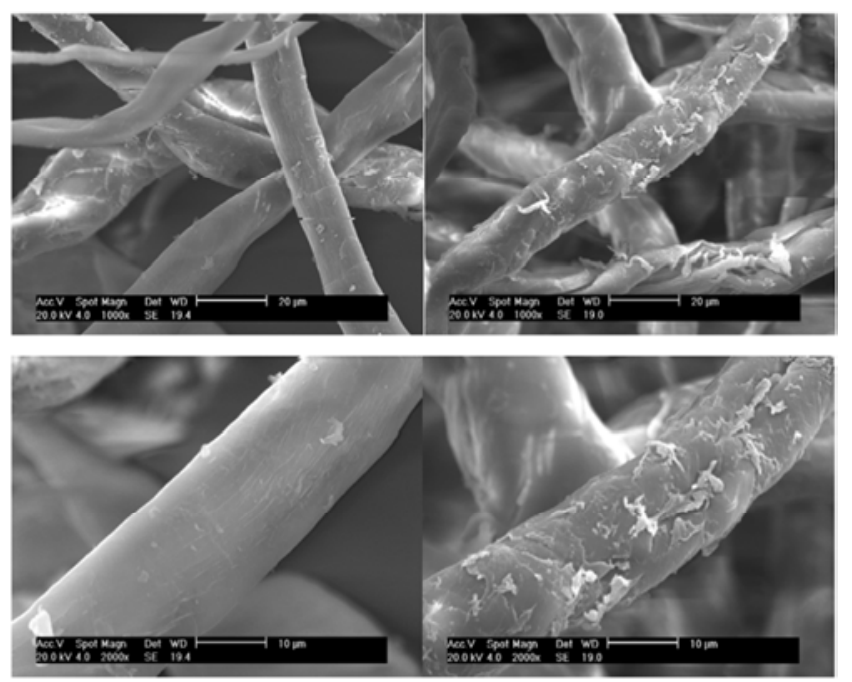

Figure 10. SEM images of the cellulose microcrystals before and after pretreatment.

\subsubsection{The Explanation to the Dosage of Chlorosulfonic Acid}

The determination of degree of substitution for cellulose sulfate by sulfuric acid was shown in the following description. The degree of substitution (DSs) by sulfuric acid is the number of hydroxide radicals in the molecule chain of cellulose that has been substituted by sulfate esters. The content of sulfur can be measured by the plasma emission spectrum according to the following equation:

$$
\mathrm{DSs}=\frac{\mathrm{M}[\mathrm{AGU}] \times \mathrm{S} \%}{\mathrm{Ms} \times 100 \%-\mathrm{S} \% \times\left(\mathrm{M}\left[\mathrm{SO}_{3} \mathrm{Na}\right]-1\right)}=\frac{162 \mathrm{~S} \%}{32-102 \mathrm{~S} \%}
$$

$\mathrm{M}$ [AGU] is the molar mass of glucose unit. $\mathrm{M}_{\mathrm{S}}$ is the molar mass of sulfur. $\mathrm{M}\left[\mathrm{SO}_{3} \mathrm{Na}\right]$ is the molar mass of the substitution group.

The degree of substitution is greatly enhanced as the amount of chlorosulfonic acid increases when chlorosulfonic acid/ AGU unit is 1.5-2. The degree of substitution is only slightly enhanced when chlorosulfonic acid/ AGU unit is 2-3. However, the degree of substitution is decreased when chlorosulfonic acid/ AGU unit is greater than 3.5. Considering the equilibrium value of hydrophilic oleophilic surfactants, the reaction condition was set when chlorosulfonic acid/ AGU unit is less than 1.5.

\subsubsection{The Type and Morphology of Cellulose}

The degree of substitution for microcrystal sodium cellulose sulfate is higher than that of linter sodium cellulose sulfate. Firstly, microcrystal cellulose is the polymerization product of natural plant cellulose hydrolyzed in the condition of weak acid. The microcrystal cellulose is obtained by degradation under acid conditions. The structure is damaged at a certain degree as shown in the SEM images. Thus, the accessibility is improved. Secondly, the degree of polymerization for microcrystal cellulose used in this experiment is much less than that of linter cellulose. It can also cause the improvement of degree of substitution.

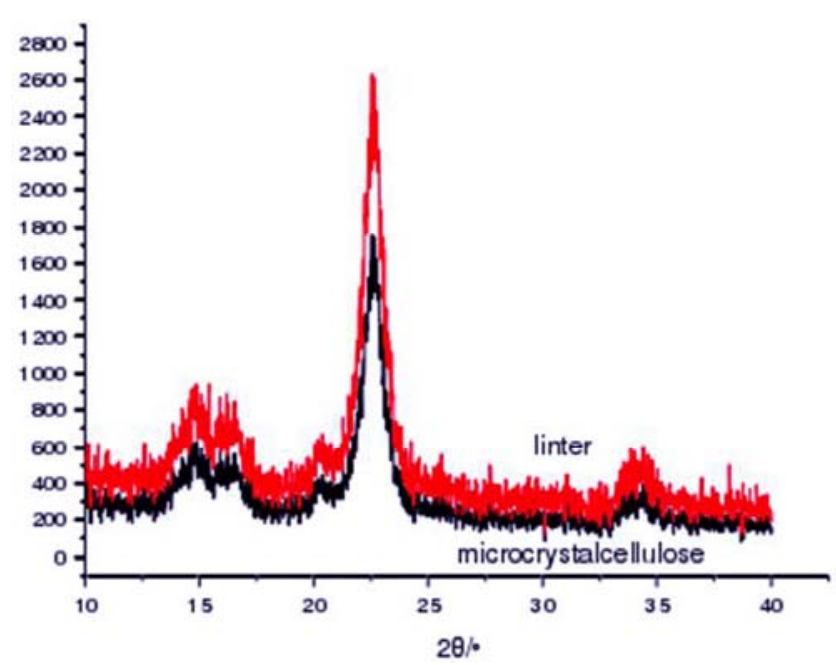

Figure 11. The degree of substitution-20 curves between microcrystal cellulose and linter cellulose which may predict the differences in both structures.

\subsection{The Measurement of Viscosity Behavior for Cellulose Sulfate}

\subsubsection{Solubility}

The equation for calculation of solubility is shown below:

$$
\mathrm{S}=\left(\mathrm{m}_{[\text {total] }}-\mathrm{m}_{[\text {ins }}\right) / \mathrm{m}_{[\text {water] }}
$$

Table 2. Solubility dependence on temperature and degree of substitution.

\begin{tabular}{llll}
\hline T/ ${ }^{\circ} \mathbf{C ~ D S}$ & $\mathbf{2 5}$ & $\mathbf{5 0}$ & $\mathbf{8 0}$ \\
\hline 0.21 & 0.14 & 0.26 & 0.30 \\
0.56 & 0.27 & 0.37 & 0.44 \\
0.99 & 0.52 & 0.87 & 0.90 \\
\hline
\end{tabular}



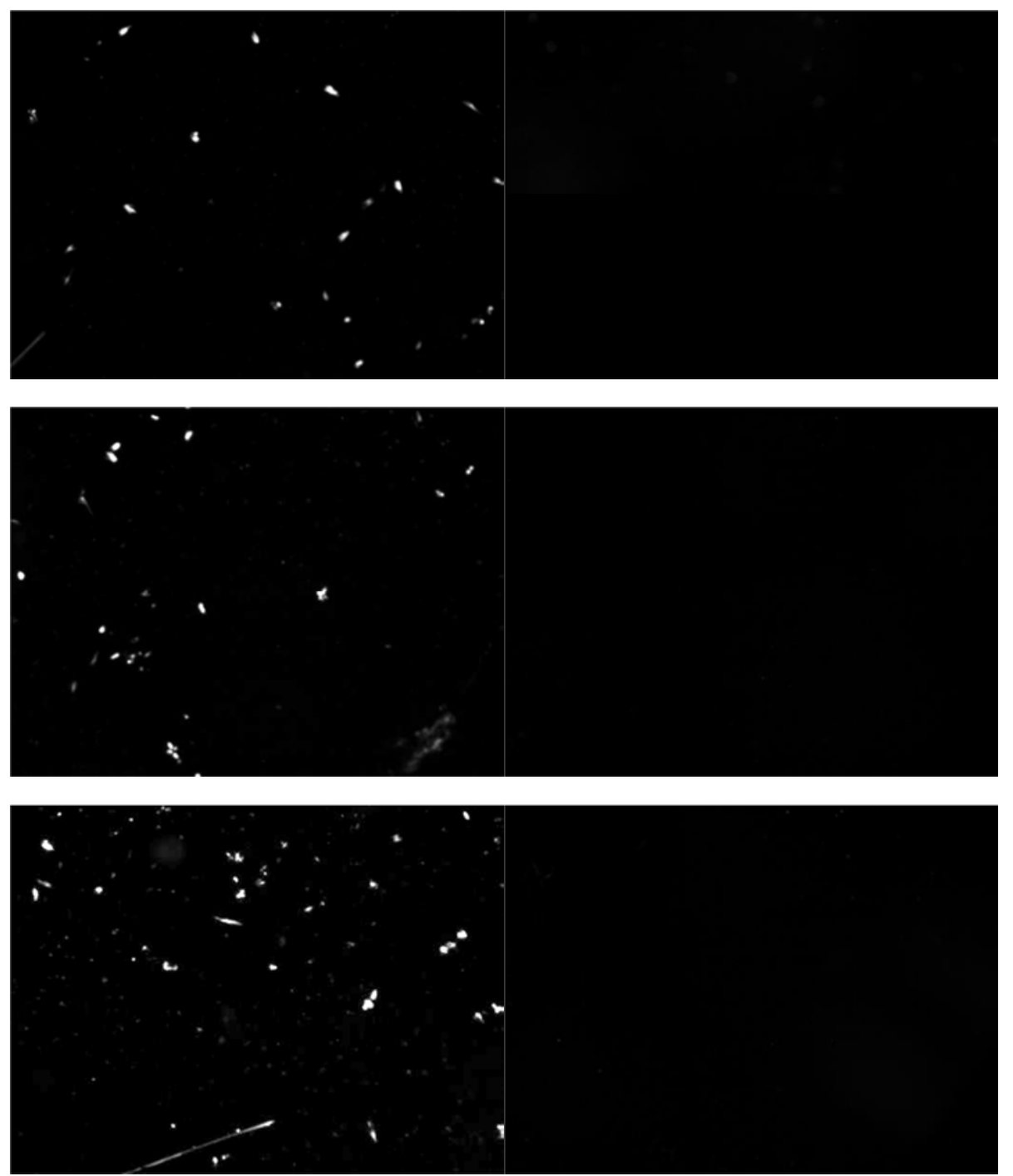

Figure 12. The polarizing microscopic image for the solution of cellulose sulfate ( $D S=0.21,0.56$ and 0.99 from top to down), left $=$ before dissolution, right $=$ after dissolution.

The solubility of cellulose can be improved by esterification with sulfuric acid. On one hand, it is because the introduction of negatively charged groups can break some hydrogen bonds and enlarge the intermolecular distance. On the other hand, sulfate group, as a hydrophilic radical, can form strong solvation when contacting with solvents, resulting in increased solubility. Due to the damage of structure induced from the sulfate groups, cellulose possesses lower crystallinity than cellulose, leading to better solubility. As shown in the polarizing microscopic images, cellulose sulfate displays a more apparent amorphous structure as the increase of degree of substitution. Less crystal structure is observed when the degree of substitution increases. As the dissolution process conducting, the crystal structure becomes less and less, until the image becomes completely dark, indicating the complete dissolution of cellulose sulfate. The solubility can be obtained from the amount of sample dissolved in water. Cellulose is insoluble in water, while cellulose possesses a high solubility in water. As shown in Figure 2, the solubility of cellulose sulfate increases as the degree of substitution by sulfate groups increases. The main reason is that the dissolution of cellulose sulfate is the process of sulfate groups being solvated by solvent when the transfer of radicals, broken of structure of cellulose and diffusion of solvents to cellulose sulfate molecules happens. Thus, cellulose sulfate with a large degree of substitution has small molecular weight, lower crystallinity, disturbed molecule arrangement and more exposed hydrophilic groups, endowing it with a high solubility. The solubility also gets higher when temperature goes up due to the fact that high temperature and vigorous stirring can promote the destruction of cellulose structure. According to the DSC curve, the cellulose with high degree of substitution is more likely to melt as the temperature increases. The high temperature benefits the molecular motion and molecular collision between water and cellulose sulfate, resulting in higher solubility.

\subsubsection{Macro Morphology and Film-forming}

The products are transparent and possess high water solubility. It became transparent after washed ethanol and existed as small colloidal particles after dried. 

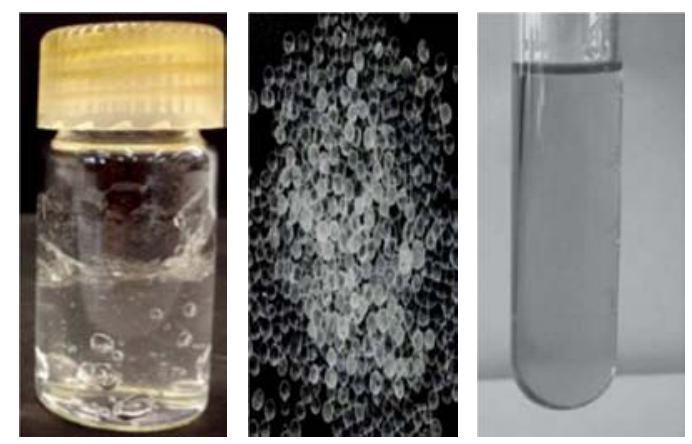

Figure 13. The photos of 3 samples which respectively is, left: products washed by ethanol, middle: dried sample, right: solution of the products.

The solution of sodium sulfate was cladded in polyethylene film, vacuum dried for $15 \mathrm{~h}$. The cellulose sulfate film was obtained and weighed after the removal of watch glass and polyethylene film.

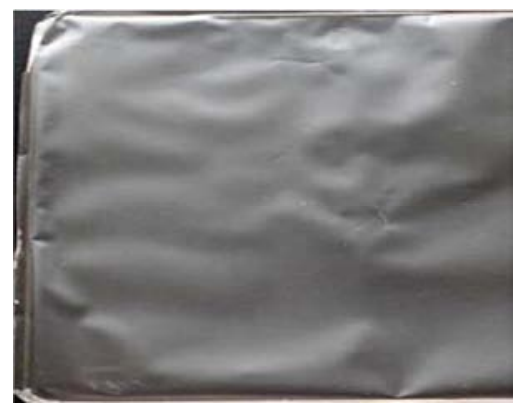

Figure 14. A photograph showing the appearance of the CS film.

The weight of the film is $0.436 \mathrm{~g}$. It is uniform, transparent and possesses a certain degree of flexibility and transparency.

\subsubsection{The Influence Factors of Viscosity (Inorganic Additives)}

The introduction of inorganic molecules can greatly improve the viscosity of the system. Cellulose sulfate possesses great consistency with inorganic materials. The development of composite material can be a new researching subject.

\section{Influence of Addictives on NACS Aqueous Solution}

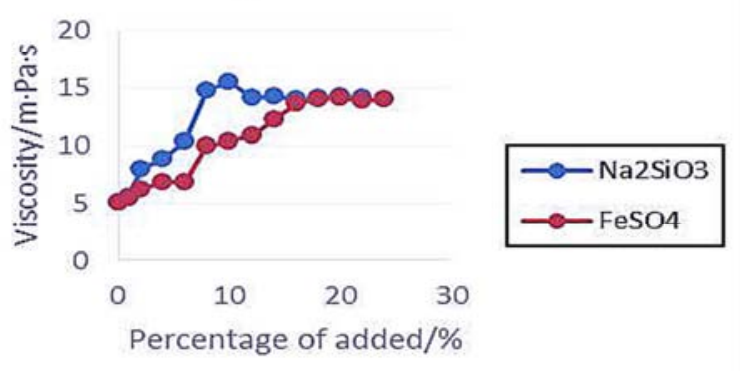

Figure 15. The viscosity-percentage of additon curve, showing the influence on the viscosity of $\mathrm{NaCS}$ aqueous solutions by additives $\mathrm{FeSO}_{4}$ or $\mathrm{NaSiO}_{3}$.

\subsubsection{Measurement of the Surface Tension}

The surface tension of the samples stabilized at 45-55 $\mathrm{mN} / \mathrm{m}$. The value first decreased and then stabilized as the concentration of surfactant increased. It indicates that polymers with strong hydrophilia possess strong capability for absorption of water molecules. However, when there is enough water in the system, the hydrophobic chain in the molecule will excrete and form a cavity system inside the polymer, resulting in rapid decrease of surface tension. When the concentration of solution reaches the critical concentration, the hydrophobic groups will associate into micelles. After that, the surface tension tends to be stable.

\subsection{The Productization Measurement for the Cellulose Sulfate Adhesive Composition}

\subsubsection{Measurement for the Sulfated Products}

The tensile shear strength of wood board with samples glued at single side.

Table 3. The tensile shear strength of different samples.

\begin{tabular}{llllll}
\hline measurements & $\mathbf{1}$ & $\mathbf{2}$ & $\mathbf{3}$ & $\mathbf{4}$ & $\mathbf{5}$ \\
\hline polyurethane & 0.465 & 0.477 & 0.432 & 0.534 & 0.512 \\
polyvinyl alcohol & 0.388 & 0.256 & 0.319 & 0.349 & 0.307 \\
NaCS & 0.489 & 0.435 & 0.502 & 0.441 & 0.498 \\
\hline
\end{tabular}

The tensile shear strength of polyurethane is $0.35-0.65$ according to the reference. The bonding strength of polyvinyl alcohol is rather weak. Thus, the results are reliable. The tensile shear strength of cellulose sulfate under certain circumstance is objective.

\subsubsection{The Influence of $\mathrm{pH}$}

Table 4. Measurement of tensile shear strength/MPa of 3 kinds of materials in different acidity.

\begin{tabular}{lllllll}
\hline $\mathbf{p H}$ & $\mathbf{3}$ & $\mathbf{4}$ & $\mathbf{5}$ & $\mathbf{6}$ & $\mathbf{7}$ & $\mathbf{8}$ \\
\hline Wood board & 0.334 & 0.548 & 0.683 & 0.462 & 0.436 & 0.351 \\
Steel plate & 0.285 & 0.452 & 0.518 & 0.612 & 0.547 & 0.420 \\
glass pane & 0.432 & 0.602 & 0.665 & 0.769 & 0.754 & 0.730 \\
\hline
\end{tabular}

The solution of sodium cellulose sulfate is acidic when $\mathrm{pH}<5$. The sodium cellulose sulfate molecule chain will break due to the hydrolysis of glucose unit in the molecule, resulting in decreased viscosity. Sodium cellulose sulfate molecules tend to exist as liner anions when $\mathrm{pH}>5$. The strong mutual exclusion will greatly influence its bonding energy, leading to decreased viscosity. The samples possess the highest tensile shear strength at $\mathrm{pH}$ from 5 to 6 (glass pane: $0.769 \mathrm{Mpa}$, steel plate: $0.612 \mathrm{Mpa}$, wood board: $0.683 \mathrm{Mpa}$ ). The excellent tensile shear strength may be associated with the existence of sulfate groups in the sodium cellulose sulfate, which can weak the exclusion between anions, suppress the hydrolysis of cellulose and form strong hydrogen bond with the molecules at the surface of the material. The exclusion effect can be reduced under acidic conditions. But it will also cause the degradation of products and the decrease of viscosity, thus benefiting the formation of hydrogen bond between molecules and influence its combination with substrate. The binding force will decrease due to the exclusion of anions. It can also influence the combination with subtract and cause the degradation of products. 


\subsubsection{The Influence of the Degree of Esterification}

The degree of esterification was determined by the degree of substitution for groups at the side chain of the molecules.

Table 5. Measurement of tensile shear strength/MPa of 3 kinds of materials in different degree of esterification (DS).

\begin{tabular}{|c|c|c|c|c|c|c|c|c|}
\hline DS & 0.4 & 0.6 & 0.8 & 1.0 & 1.2 & 1.4 & 1.6 & 1.8 \\
\hline Wood board & 0.309 & 0.551 & 0.791 & 0.887 & 0.722 & 0.683 & 0.607 & 0.532 \\
\hline Steel plate & 0.362 & 0.713 & 0.860 & 1.054 & 0.936 & 0.879 & 0.692 & 0.515 \\
\hline glass pane & 0.451 & 0.690 & 1.058 & 1.162 & 0.935 & 0.836 & 0.748 & 0.492 \\
\hline
\end{tabular}

The dissolution will happen only when the degree of substitution of esterification is greater than 0.34 . Thus the data range for degree of substitution starts form 0.4. The adhesive strength reaches a maximum when DS is 1.0. Excessive substitution can cause aggregation of sulfonic acid groups and accumulation of negative charges. The repulsive force will result in decrease of adhesive strength.

\subsubsection{The influence of $\mathrm{Mg}^{2+}, \mathrm{Sn}^{2+}$ and Carboxymethyl Dextran at $\mathrm{pH}=6$}

The adhesive strength of cellulose sulfate first increases and then decreases as the amount of magnesium ions increases. The adhesive strength of the three substrates reaches maximum all at $2 \%$. Due to the fact that the neat network structure formed with magnesium ions and polymer anions weakens the repulsive force between the anions, it can enhance the tensile shear strength of the materials that attached to sodium cellulose sulfate. The tensile shear strength decreases when the additive amount exceeded $2 \%$. It may be associated to that excessive magnesium ions will induce repulsive interaction of positive ions. Thus, the tensile shear strength was reduced.

The influence of tin ions is not as remarkable as magnesium ions. A relaxation of activity is formed when the active hydroxide radicals contact with heavy metal ions. The change in tensile shear strength is strongly suppressed. The phenomenon is profound in any board materials [16].

Carboxymethyl dextran is a high molecular polymer with positive charge. It can form strong electrostatic attraction with cellulose sulfate and greatly enhance the adhesive strength of biomass substrate, such as wood board. Little amount of carboxymethyl dextran can induce great enhancement to the tensile shear strength. But the effect is greatly suppressed at steel plate and glass pane. It can be associated to the accumulated positive charge and weakened attraction.

Table 6. Measurement of tensile shear strength/MPa of 3 kinds of materials in different categories of solvents.

\begin{tabular}{|c|c|c|c|c|c|c|c|c|c|c|}
\hline & \multirow{2}{*}{ Blank } & \multicolumn{3}{|c|}{ magnesium sulfate } & \multicolumn{3}{|c|}{ stannous mono-sulfate $\left(\mathrm{H}^{+}\right)$} & \multicolumn{3}{|c|}{ carboxymethyl dextran } \\
\hline & & $1 \%$ & $2 \%$ & $3 \%$ & $1 \%$ & $2 \%$ & $3 \%$ & $1 \%$ & $2 \%$ & $3 \%$ \\
\hline wood board & 0.449 & 0.544 & 0.745 & 0.712 & 0.457 & 0.461 & 0.460 & 0.534 & 0.587 & 0.892 \\
\hline steel plate & 0.618 & 0.678 & 0.699 & 0.637 & 0.687 & 0.673 & 0.679 & 0.612 & 0.609 & 0.576 \\
\hline glass pane & 0.757 & 0.895 & 0.972 & 0.951 & 0.772 & 0.765 & 0.768 & 0.738 & 0.714 & 0.707 \\
\hline
\end{tabular}

\section{Conclusion}

In this paper, we explicitly identify the microlevel colloidal and adhesion mechanism based on the synthesis and optimal design of a new type of environmentally friendly, cellulose-based adhesive. It is proved to have a strong viscosity and adhesion strength and expected to be translated into more real-life applications in industry.

First, we fabricated a new form of cellulose sulfate with a certain mode of substitution, which demonstrated outstanding adhesive-related performance. For more tunable enhancement of these performance, we measured, analyzed, and partly quantified the solubility, film-forming trait and viscosity and investigated their relations of external conditions. In the industrial test, the product revealed a greater adhesion capability with the cross link with substrates or formation of non-molecular interactions (e.g. hydrogen bonding, coordinated coupling).

The cellulose-sulfate-based adhesive is expected to have great potential in industrial functionalization. Its adhesive-related behaviour is more likely to be precisely controlled based on structure-property analysis, particularly the connections among morphological characterization, gel-related colloidal properties and hydrodynamic analysis.

\section{Acknowledgements}

This project is fully funded by AC R \& D Team of Xiamen University. It is also well supported by Tencent Charity Foundation, China.

\section{References}

[1] Zhang J. M. and Zhang J. (2010) Advanced Functional Materials Based on Cellulose. Acta Polymerica Sinica (12): 1376-1378.

[2] Pierre A. C. and Pajonk G. M. (2002) Chemistry of Aerogels and Their Applications. Chemical Reviews 102 (11): 4243-4266.

[3] Edgar K. J., Buchanan C. M., Debenham J. S., Rundquist P. A., Seiler B. D., Shelton M. C. and Tindall D. (2001) Advance in Cellulose Ester Performance and Application. Progress in Polymer Science 26 (9): 1605-1688. 
[4] Zhang J., Zhang L. M. and Li Z. M. (2000) Synthesis and Aqueous Solution Properties of Hydrophobically Modified Graft Copolymer of Sodium Carboxymethyl Cellulose with Acrylamide and Dimethyloctyl (2-methacryloxyethyl) Ammonium Bromide. Journal of Applied Polymer Science 78 (3): $537-542$.

[5] Zhang L. M. (1999) Inhibitive Properties of Amphoteric, Water-Soluble Cellulosic Polymers on Bentonite Swelling. Colloid \& Polymer Science 277 (2-3): 282-284.

[6] Holme I. (2010) Advanced Ideas for Cellulosics. International Dyer 195 (9): 9-11.

[7] Yamamoto I., Takayama K., Honma K., Gonda T., Matsuzaki K., Hatanaka K., Uryu T., Yoshida O., Nakashima H. and Yamamoto N. (1990) Synthesis, Structure and Antiviral Activity of Sulfates of Cellulose and Its Branched Derivatives. Carbohydrate Polymers 14 (1): 53-63.

[8] Appaw C., Gilbert R. D. and Khan S. A. (2007) Viscoelastic Behavior of Cellulose Acetate in a Mixed Solvent System. Biomacromolecules 8 (5): 1541-1547.

[9] Nyström B., Walderhaug H., Hansen F. K. and Lindman B. (1995) Rheological Behavior during Thermoreversible Gelation of Aqueous Mixtures of Ethyl (Hydroxyethyl) Cellulose and Surfactants. Langmuir 11 (3): 750-757.

[10] Zhou J., Xu Y., Wang X., Qin Y. and Zhang L. (2008) Microstructure and Aggregation Behavior of Methylcelluloses Prepared in NaOH/Urea Aqueous Solutions. Carbohydrate Polymers 74 (4): 901-906.

[11] Lv X., Li L., Lin Z. and Cui S. (2011) Formation Mechanism of Ionic Liquid Regenerated Cellulose Hydrogel and Its Application in Gel Electrophoresis. Acta Polymerica Sinica 011 (9): 1026-1032.

[12] Kavanagh G. M. and Ross-Murphy S. B. (1998) Rheological Characterization of Polymer Gels. Progress in Polymer Science 23 (3): 533-562.

[13] Fan L., Zhou X., Wu P., Xie W., Zheng H., Tan W., Liu S. and Li Q. (2014) Preparation of Carboxymethyl Cellulose Sulfates and its Application as Anticoagulant and Wound dressing. International Journal of Biological Macromolecules 66 (0): 245-253.

[14] Gauche C. and Felisberti M. I. (2019) Colloidal Behavior of Cellulose Nanocrystals Grafted with Poly (2-alkyl-2-oxazoline)s. ACS Omega 4 (7): 11893-11905.

[15] Sandak A., Jaszczur A., Sandak J. and Modzelewska I. (2015) Near Infrared Assessment of Biodegradability and Mechanical Properties of Paper Made of Cellulose Sulfate Bleached Coniferous Pulp with Addition of Cationic Starch and Resinous Adhesive. International Biodeterioration \& Biodegradation 97: 31-39.

[16] Lai Y. L., Zhang H., Sugano Y., Xie H. and Kallio P. (2019) Correlation of Surface Morphology and Interfacial Adhesive Behavior between Cellulose Surfaces: Quantitative Measurements in Peak-Force Mode with the Colloidal Probe Technique. Langmuir 35 (22): 7312-7321.

[17] Yao S. (2000) An Improved Process for the Preparation of Sodium Cellulose Sulphate. Chemical Engineering Journal 78 (2-3): 199-204. 